\title{
Bases para \\ desenvolvimento de um projeto cromático como intervençōes de melhoria em áreas urbanas degeneradas usando tecnologias de Realidade Virtual e Realidade Aumentada
}

Anamaria Amaral Rezende G é graduada, mestre e doutora em Arquitetura e Design pela FAU-USP, obteve também a especialização em Comunicação e Educação pela Universidade Anhembi Morumbi (1998). Pesquisa, escreve e leciona sobre cor aplicada ao design. É professora em cursos de Arquitetura e Design há mais de 20 anos e desenvolveu uma instalação 3D interativa e imersiva durante seu doutorado-sanduíche como bolsista Capes-Fulbright na Stamps School of Arts and Design da Universidade de Michigan (2013-2014). Tem larga experiência em projetos de Arquitetura Comercial, Design Gráfico e Design Ambiental.

<ana.rezende@anhembi.br> ORCID: 0000-0001-5268-8298
Resumo Podem as tecnologias de Realidade Virtual e Realidade Aumentada contribuir em projetos de design urbano como recursos de visualização e de verificação de seus resultados, tornando-os acessíveis e de baixo custo? Este projeto, em fase inicial, estuda a cor enquanto forma de expressão identitária no espaço público urbano e procura identificar, entender e apreender as maneiras como esse elemento pode intervir nos processos de modificação da cidade de forma a auxiliar a ressignificação e a reapropriação de uma identidade local. Focando-se em espaços públicos de áreas urbanas degeneradas, ele contempla a elaboração de um projeto cromático experimental em uma comunidade periférica da cidade de São Paulo, envolvendo os atores sociais da mesma, bem como estudantes e voluntários, com o objetivo de determinar um método para a elaboração de projetos cromáticos de intervenção urbana apoiados pela tecnologia.

Palavras chave Cor, Design urbano, Identidade, Realidade virtual, Placemaking Criativo. 
Clice de Toledo Sanjar Mazzilli Professora Associada da Faculdade de Arquitetura e Urbanismo da Universidade de São Paulo, onde leciona desde 2001. Atualmente coordena o Programa de Pós-graduação em Design e o Programa de Duplo Diploma em Design entre a FAU-USP e o Politecnico di Milano (Scuola del Design). Obteve Bolsa Sanduiche da CAPES para estágio de pesquisa na Facoltà di Architettura del Politecnico di Torino (1990) e também realizou um Pós-doutorado na Universidad Politecnica de Madrid (2010) com bolsa da Fundación Carolina. No período de 1997 a 2006 lecionou no Instituto de Artes da UNESP. Orienta e desenvolve pesquisas sobre processos experimentais e linguagem em Design Visual, e sobre indentidade cromática do ambiente urbano. Tem experiência profissional nos campos do Design Ambiental e da Comunicação Visual. <clice@usp.br> ORCID: 0000-0002-6903-9099

\section{Foundations for the development of a chromatic project as improvement inter- ventions in urban decayed areas using Virtual Reality and Augmented Reality technologies}

Abstract Can Virtual Reality and Augmented Reality technologies contribute as visualization and result verification resources in urban design projects, making them both accessible and affordable? This project, which is still in its initial stages, studies color as a means for expressing identity in public urban spaces and aims to identify, understand and apprehend the ways in which this element can intervene in the city's modification processes so as to help in the resignification and reappropriation of local identities. Focusing in public spaces of urban decayed areas, it includes the elaboration of an experimental chromatic project in a suburban community of the city of São Paulo, with the participation of its social actors, as well as of students and volunteers, and with the goal of determining a method for the elaboration of chromatic projects for urban intervention supported by technology.

Keywords Color, Urban Design, Identity, Virtual Reality, Creative Placemaking.

Bases para el desarrollo de un proyecto cromático como intervenciones de mejora en áreas urbanas deterioradas utilizando tecnologias de Realidad Virtual y Realidad Aumentada

Resumen ¿Pueden las tecnologías de Realidad Virtual y Realidad Aumentada contribuir con proyectos de diseño urbano como recursos de visualización y de verificación de sus resultados, tornándolos accesibles y de bajo costo? Este proyecto, que se encuentra en fase inicial, investiga el color en cuanto forma de expresión identitaria en el espacio público urbano y busca identificar, comprender y aprehender las maneras como tal elemento puede participar en los procesos de modificación de la ciudad a fin de ayudar la resignificación y la reapropiación de una identidad local. Con foco en los espacios públicos de áreas urbanas deterioradas, él contempla la elaboración de un proyecto cromático experimental en una comunidad periférica de la ciudad de São Paulo, involucrando sus actores sociales, así como los estudiantes y los voluntarios, con el objetivo de determinar un método para la elaboración de proyectos cromáticos de intervención urbana ayudados por la tecnología.

Palabras clave Color, Diseño urbano, Identidad, Realidad virtual, Placemaking creativo. 
Bases para desenvolvimento de um projeto cromático como intervenções de melhoria em áreas urbanas degeneradas usando tecnologias de Realidade

\section{Introdução}

A questão "cor ambiental urbana" enquanto elemento de expressão da identidade de um lugar - capaz de contribuir para conferir ou resgatar o senso de comunidade e senso de tal lugar e, consequentemente, o senso de pertencimento de uma comunidade degenerada e abandonada - é o ponto central deste projeto. $\mathrm{E}$, com a constatação de que o ensino da cor em cursos de Arquitetura e Urbanismo, bem como de Design - no Brasil principalmente - é anêmico, insuficiente, ou mesmo inexistente, vislumbrou-se a necessidade da elaboração de bases para a criação de projetos cromáticos que atuem como ações interventoras em espaços públicos, do tipo Placemaking Criativo e Tático.

Propõe-se o desenvolvimento de um projeto experimental em área urbana com grande potencial para melhorias, obtendo-se o envolvimento e a participação da comunidade e objetivando uma intervenção cromática com artistas locais. $O$ projeto cromático da intervenção (e o projeto como um todo) será posto a votação popular utilizando-se Realidade Virtual (VR, ou 3D imersivo) e Realidade Aumentada (AR). Prevê-se, assim, uma verificação (um teste de baixo custo) do projeto e do uso da cor enquanto elemento gerador de identidade local por meio da utilização inovadora de tal tecnologia. Munidos desse diferencial, a pesquisa e o projeto experimental pretendem, ao final, ter respondido à seguinte questão: podem as tecnologias de Realidade Virtual e Realidade Aumentada contribuir em ações de design urbano?

\section{Cenário}

O estabelecimento de espaços públicos inclusivos e funcionais até 2030 é uma preocupação da Nova Agenda Urbana da ONU-Habitat, publicada em 2016. O programa da ONU para assentamentos humanos, criado em 1978 para cuidar de questões relacionadas ao crescimento urbano, afirma que, em 2050, dois terços (2/3) da população mundial viverá em cidades. Diante disso, o documento demonstra preocupação com as exacerbadas desigualdades econômicas e sociais: "A falta de espaços públicos de qualidade reduz a qualidade de vida urbana, aumentando a criminalidade, as tensões sociais, a saúde e o congestionamento" (UN-Habitat, 2016 grifo nosso).

\footnotetext{
O que define o caráter de uma cidade são os espaços públicos. O valor dos ativos privados do espaço não são os ativos em si, mas o conjunto de ativos. O valor do bem público afeta o valor do bem privado. Precisamos mostrar todos os dias que os espaços públicos são os ativos para a cidade. (MATHEU apud ONU, 2012, p. 2)
} 
Ainda sobre os espaços públicos, Joan Clos, em mensagem escrita para o World Habitat Day+ Public Spaces for All, alega que:

\footnotetext{
O espaço público oferece espaço para interação social e cultural e pode promover um sentimento de pertencimento e orgulho em uma área... Esses espaços moldam a identidade cultural de uma área, fazem parte de seu caráter único e proporcionam um senso de lugar para as comunidades locais. (MATHEU, 2015)
}

Espaços degenerados ou que sofreram grande degradação podem se transformar em novos lugares. Há diversos exemplos de intervenções de transformação sobre a realidade existente que não constroem novas obras, mas que propõem uma imagem radicalmente nova e de grande impacto visual, comunicativo e emocional, recorrendo à expressividade da cor para reforçar o efeito dinâmico e, por vezes, interativo das superfícies (BOERI, 2016).

\section{Cor (como significado gerador de expressão), identidade e ações de Placemaking}

Arquiteta e pesquisadora do laboratório de cor da Politécnica de Milão (POLIMI, Itália), Cristina Boeri defende em sua tese (Color Loci Placemaking, 2017) que a cor confere reconhecimento e senso de pertencimento à imagem da cidade enquanto elemento de linguagem e expressão. Apesar do viés histórico de suas análises e fundamentações, nota-se que a cor está carregada de significados universais e que ela incrementa uma experiência e promove um sentido de lugar, além de estimular atmosferas positivas e fruitivas para quem habita e para quem se relaciona de alguma maneira com áreas que sofreram uma intervenção cromática resultante de uma ação de Placemaking Criativo. Assim, o uso da cor relaciona um contexto físico-espacial com um histórico-temporal, ambos refletidos na identidade da cidade.

\section{Cor em design urbano}

A cor ambiental como fenômeno psicofísico é percebida conjuntamente a variáveis tais como: texturas, contrastes cromáticos, o material e a aparência dos limites, a posição do observador, o modo e velocidade de deslocamento, entre outros. Acrescido a isso, assume-se que a função primordial das cores é conferir significações, a fim de estabelecer uma relação comunicativa entre o indivíduo e o ambiente físico. Consequentemente, a 
experiência cromática depende da interação quantitativa e qualitativa de diferentes componentes e contextos que nela participam (BALIAN; KENNY; SUAREZ et SUEZ, 2018, p. 4).

A cor no espaço urbano - um dos componentes estruturais da linguagem da cidade - corrobora com as ansiedades, satisfações e desejos do homem e de sua experiência urbana e com a construção de um sentido de lugar (SUAREZ, 2018). Defendemos que a expressão cromática é um movimento e a cor ambiental urbana é parte ativa das mudanças produzidas na vida da sociedade, as quais se manifestam de maneiras distintas nos espaços urbanos. Um projeto cromático urbano tem seu valor a partir do instante que nasce da percepção e vivência cotidiana dos moradores e reflete a identidade do lugar - o que gera a sensação de pertencimento.

Cristina Boeri (2017) defende que a identidade cromática de uma cidade é um sistema complexo de componentes materiais e imateriais. Uma complexidade que oferece diferentes modalidades e possibilidades de intervenção e que requer abertura e flexibilidade constantes para redefinir os termos nos quais se irá operar. É preciso estabelecer, por exemplo, se a ação terá um caráter contínuo e/ou conservador, no que diz respeito ao componente e material cromático, e também onde intervir, com ações focadas em apoiar as transformações e os novos significados do espaço urbano.

Porém, a percepção das cores em um dado lugar não depende exclusivamente do contexto físico-espacial; ela é também influenciada pelos estados emocionais e pela condição cultural da pessoa que a percebe, ou seja, o contexto histórico e cultural. Portanto, a ação da cor enquanto forma de expressão é fundamental, uma vez que seu caráter comunicacional tem se estendido por novos modos de produção e sistemas de resolução física e digital, aumentando sua capacidade de informar, sugerir, desenvolver associações sinestésicas e criar atmosferas. Assim, ela induz à experiência vivencial na cidade e à consequente promoção da vida urbana, pois é fundamental para reforçar sua identidade, seu desenho e construção (Dario at al., 2018).

Este projeto, que está em fase inicial de desenvolvimento, visa estabelecer as bases para o desenvolvimento de um projeto cromático associado ao design urbano e a ações de Placemaking Tátil e Criativo. Pretende também analisar a possibilidade e viabilidade de transformar espaços públicos em zonas degradadas por meio de projetos atemporais, sustentáveis e criativos que promovam o senso de lugar e traduzam o senso de pertencimento, ou seja, que garantam mais do que uma solução estética, conferindo um valor emblemático, social e identitário.

Abordaremos a seguir como essas ações associadas ao uso da cor na paisagem urbana, em suas definições, articular-se-ão no projeto aqui proposto. Mas, antes, vamos expor os significados com os quais são utilizados os conceitos de Placemaking e Placemaking Criativo. 
Bases para desenvolvimento de um projeto cromático como intervenções de melhoria em áreas urbanas degeneradas usando tecnologias de Realidade Virtual e Realidade Aumentada

\section{Placemaking}

Ações de Placemaking vêm se consolidando como uma forma de melhorar a qualidade de vida em vários lugares de um bairro e, por extensão, em toda a região em que esses lugares estão localizados. No entanto, a variedade de usos do termo nos leva a esclarecer algumas diferenças para, assim, não incorrer no risco de diluir o valor do conceito e atenuar a relevância deste projeto.

Placemaking, como significado amplo, é um termo da geografia cultural e está intimamente associado ao "senso de lugar". É a maneira como um grupo cultural imprime seus valores, percepções, memórias e tradições em uma paisagem e, dessa forma, fornece sentido ao espaço geográfico (2013 apud LEW, 2017, p. 2). Wyckoff (2014), em Definition of placemaking: Four Different Types, define-o como "o processo de criar lugares de qualidade em que as pessoas querem viver, trabalhar, brincar e aprender"(WYCKOFF, 2014, p. 2). Ele reforça ainda que se trata de um processo, um meio para um fim; o fim é a "criação de lugares" de qualidade.

Todavia, as pessoas somente conhecem e entendem o que são lugares de qualidade quando os ocupam e, claramente, é mais desafiador descrever tais características abstratamente. A preocupação reside em criar lugares com os quais as pessoas se importem e onde queiram estar; ou seja, pontos que possuam um forte senso de lugar.

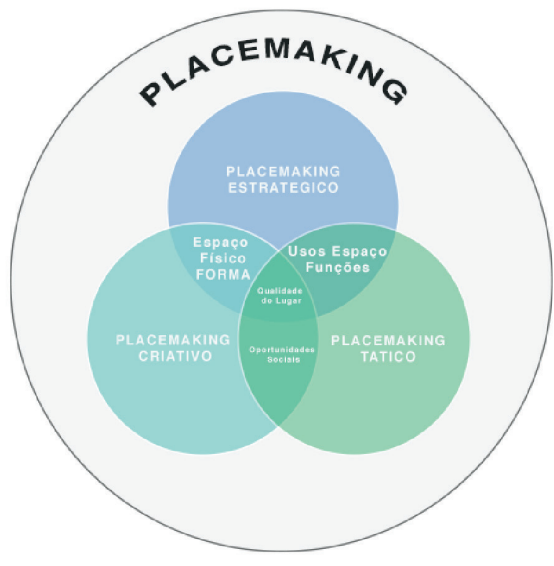

Fig 1. Quatro tipos de Placemaking segundo Mark A. Wyckoff Fonte: Ilustração do autor.

\begin{abstract}
O lugar é mais do que apenas um local ou um ponto em um mapa. Um senso de lugar é uma coleção única de qualidades e características - visual, cultural, social e ambiental - que dá sentido a um local. Senso de lugar é o que torna um local (por exemplo, sua cidade natal) diferente de outro local (por exemplo, minha cidade natal), mas o senso de lugar também é o que faz com que valha a pena se preocupar com nosso ambiente físico. (McMAHON, 2010, p.16)
\end{abstract}

São locais ativos, únicos, interessantes, visualmente atraentes, muitas vezes com arte pública e atividades criativas. São considerados amigáveis, seguros e onde se pode caminhar, com usos mistos (WYCKOFF, 2014).

Wyckoff define ainda Placemaking - em sua acepção mais ampla e universal - como sendo resultado da combinação de, basicamente, três tipos de ações (Figura 1): Placemaking Estratégico, Placemaking Criativo e Placemaking Tático.

Como este projeto visa relacionar seu projeto cromático ao resgate da identidade e dos sensos de lugar e de comunidade em um espaço público, não nos aprofundaremos nas definições de Placemaking Estratégico, apresentadas esquematicamente no Quadro 1, a seguir. 
Quadro 1. Síntese das quatro definições de Placemaking segundo Wyckoff Fonte: Quadro da autora.

\begin{tabular}{|c|c|c|}
\hline OProblama & Schuge & Resulados \\
\hline \multicolumn{3}{|c|}{ Placemaking (no sentido amplo do temo) } \\
\hline $\begin{array}{l}\text { Comunidades não estão efetivamente } \\
\text { usando o espaço público para criar } \\
\text { uma comunidade vibrante e habitável } \\
\text { cujos habitantes queiram } \\
\text { viver, trabalhar, brincar ou se educar. }\end{array}$ & $\begin{array}{l}\text { Envolvimento de um público amplo e } \\
\text { interessado em revitalizar, dar novos } \\
\text { usos, criando espaços públicos, } \\
\text { utilizando técnicas de curto e longo } \\
\text { prazo basedas em princíios de design } \\
\text { social e novos princípios de design } \\
\text { urbano }\end{array}$ & $\begin{array}{l}\text { Mais Lugares de qualidade com atividade } \\
\text { de qualidade e com um forte senso de } \\
\text { lugar. Mais espaços públicos habitáveis, } \\
\text { vibrante em comunidades e regióes cujos } \\
\text { moradores, negócios, empresas e } \\
\text { visitantes se importam } \\
\text { verdadeiramente com eles. }\end{array}$ \\
\hline \multicolumn{3}{|c|}{ Placemaking Estratígoico } \\
\hline $\begin{array}{l}\text { Comunidades não são } \\
\text { competitivas em atrair e reter } \\
\text { trabalhadores especializados. }\end{array}$ & $\begin{array}{l}\text { Revitalização que aumente as escolhas } \\
\text { por habitaçōes, opçôes de transportes } \\
\text { e amenidades urbanas para } \\
\text { atrair trabalhadores especializados. }\end{array}$ & $\begin{array}{l}\text { Resultados mais rápidos em termos de } \\
\text { qualidade de vida, aumento da } \\
\text { populaçăo, diversidade, empregos, } \\
\text { renda e grau de instruçâo se comparado } \\
\text { 'as açōes de placemaking (amplo). }\end{array}$ \\
\hline \multicolumn{3}{|c|}{ Placemaking Ciativo } \\
\hline \begin{tabular}{|l|} 
Metrópolis, subúrbios e \\
pequenas cidades confrontam \\
mudanças estruturais, \\
marginalização e desenraizamento social.
\end{tabular} & $\begin{array}{l}\text { Revitalização por } \\
\text { iniciativas criativas que revivam os } \\
\text { lugares e ativem o desenvolvimento } \\
\text { econômico. }\end{array}$ & $\begin{array}{l}\text { Ganhos em habitabilidade, diversidade, } \\
\text { geraçấode empregos e renda. } \\
\text { Criação de produtos inovadores e } \\
\text { serviços para a indústria cultural. }\end{array}$ \\
\hline \multicolumn{3}{|c|}{ Placemaking Tfico } \\
\hline \begin{tabular}{|l|} 
Melhorias fisicas são caras \\
e os investidores (público/privado) são \\
relutantes em comprometer recursos \\
devido a riscos incertos \\
e resultados duvidosos. \\
\end{tabular} & $\begin{array}{l}\text { Uso de realização de testes de baixo } \\
\text { custo para avaliar a } \\
\text { eficácia da açáo proposta e apoio } \\
\text { público. }\end{array}$ & $\begin{array}{l}\text { O público e os tomadores de decisōes } \\
\text { políticas podem verificar os resultados e } \\
\text { grau de apoio para as iniciativas antes de } \\
\text { comprometer com os recursos } \\
\text { permanentes. }\end{array}$ \\
\hline
\end{tabular}

Em Tactile Placemaking, de Mike Lydon, Dan Bartman, Tony Garcia, Russ Preston e Ronald Woudstra (2012), os autores descrevem que a melhora na habitabilidade de nossas cidades geralmente começa na escala das ruas, nos quarteirões ou nos edifícios. Embora os esforços em larga escala tenham seu lugar, melhorias incrementais em pequena escala são vistas cada vez mais como uma forma de realizar investimentos mais factíveis e realísticos.

Essa abordagem permite que vários atores locais testem novos conceitos antes de assumir compromissos políticos e financeiros substanciais. Às vezes sancionadas, às vezes não, as ações são comumente chamadas de urbanismo guerrilheiro, urbanismo pop-up, reparação urbana ou urbanismo DIY (abreviação em inglês para do it yourself ou, em português, "faça você mesmo"). Segundo Eric Reymolds, líder do Urban Space Management, esse tipo de Placemaking, visto como uma estratégia de desenvolvimento local denominada Lighter, Quicker, Cheaper (LQC), produziu alguns dos espaços públicos mais bem-sucedidos do mundo com menor risco e menor custo, aproveitando a energia criativa da comunidade para gerar eficientemente novos usos e promover uma injeção de recursos em lugares em fase de transição.

Classificaremos, portanto, o projeto aqui apresentado como uma proposta experimental do tipo Soft Project (BOERI, 2017) e de transformações de superfícies (BATTISTI, 1978 apud BOERI, 2017, p. 645), e também como um projeto do tipo LQC, uma vez que ele almeja dar início, de maneira simples, a um projeto de design urbano de curto prazo e baixo custo, com envolvimento da comunidade e testando as tecnologias de VR e RA para obter tais resultados. 
Bases para desenvolvimento de um projeto cromático como intervenções de melhoria em áreas urbanas degeneradas usando tecnologias de Realidade

\section{Placemaking Criativo}

O termo Placemaking Criativo, foi utilizado pela primeira vez por Ann Markusen e Anne Gadwa ao escreverem a obra Creative Placemaking para a National Endowment for the Arts (NEA), em 2010.

(...) parcerias com os setores público, privado, sem fins lucrativos e comunitário para moldar estrategicamente as características econômicas, físicas e sociais de um lugar em torno de atividades artísticas e culturais. o placemaking criativo anima espaços públicos e privados, rejuvenesce estruturas e ruas, melhora a viabilidade dos negócios locais e a segurança pública, e reúne diversas pessoas para celebrar, inspirar e inspirar-se. (GADWA; MARKUSEN, 2010, p. 3)

Debra Webb defende a expansão da atuação do Placemaking Criativo, uma vez que "artistas desempenham um papel importante no avanço da humanidade por meio de iniciativas criativas baseadas em lugares e essa mudança não é apenas com relação a estética do lugar, mas também uma estética do pertencimento" (WEBB, 2013, p.38). Com relação à comunidade, essas ações devem fornecer oportunidades autênticas para seus membros expressarem suas relações com o ambiente físico e social.

A autora afirma ainda que os modelos atuais de Placemaking Criativo estão ligados ao ambiente construído e à revitalização urbana e defende um novo modelo, a fim de: desenvolver o senso de pertencimento do lugar; possibilitar medidas de empoderamento; utilizar o gerenciamento cultural e a aderência da comunidade como indicadores de sucesso. Ela recomenda que tal estrutura expandida inclua três componentes, a saber: Placemaking guiado por atividades de engajamento cívico que fomentem o desenvolvimento cultural; Placemaking que estimule a mudança social sistêmica e o empoderamento da juventude; e ações que articulem uma estética compartilhada de pertencimento, um senso de comunidade.

O propósito das intervenções de Placemaking refere-se, então, ao processo de criação de lugares onde as pessoas se sintam confortáveis e acolhidas, com a crença de que espaços urbanos interessantes, seguros e atraentes convidarão as pessoas a se reunirem e se conectarem para construir uma estrutura comunitária que contribua para uma maior resiliência urbana.

Ações de Placemaking podem se desenvolver de maneira formal e informal e concorda-se que, para criar uma boa ligação entre o local e a comunidade, a abordagem para o estabelecimento do senso de lugar passa pelo reconhecimento da comunidade como "especialistas" na tomada de decisões, ao invés de confiar apenas em especialistas profissionais de outros lugares. 
Isso nos leva ao capítulo três do World Cities Report 2016, e particularmente ao item 3.5, Progress Made with Respect to adequate Housing (Progresso realizado com respeito à moradia adequada), e ao subitem Upgrading poor neighbourhoods (Melhorando bairros pobres), pois eles fazem uma alusão direta à importância da participação da comunidade nas decisões de planejamento das melhorias de suas áreas, além de reforçarem que essa participação pode preservar o senso de pertencimento, assim como assegurar que os serviços propostos sejam aquilo que seus moradores desejam, valorizam e estão dispostos a cuidar. Quando essa participação ocorre desde a fase de planejamento, ela contribui para o sucesso do projeto.

Os autores McMillan e Chavis nos fornecem quatro critérios para definir a expressão "senso de comunidade", aspecto psicológico do ser humano que descreve a sensação genuína de conexão e pertencimento a um determinado grupo de pessoas.

\footnotetext{
O primeiro elemento é a afiliação, adesão (simbólico). Ele simboliza a afiliação, significa uma adesão, é o sentimento de pertencer ou de compartilhar um sentido de relacionamento pessoal. O segundo elemento é influência, um sentido de importância, sua capacidade de influenciar e ser influenciado, de fazer a diferença para um grupo e do grupo que faz a diferença aos seus membros. O terceiro elemento é o reforço: reforço que somos importantes uns para os outros, como uma integração e satisfação de necessidades. Este é o sentimento de que as necessidades dos membros serão satisfeitas e os recursos recebidos por meio de seu compromisso em participar do grupo. $O$ último elemento é a conexão emocional compartilhada, o compromisso e a crença de que os membros compartilharam e compartilharão histórias, lugares, tempo juntos e experiências semelhantes, na alegria ou na tristeza. (CHAVIS; MCMILLAN, 1986, p. 12)
}

\section{A proposta de intervenção}

$\mathrm{Na}$ crença de que "a cor pode ser hoje um importante elemento de reconhecimento, pertença e legibilidade da cidade, mas também motor de um processo social mais profundo e duradouro de transformação e reapropriação dos lugares urbanos" (BOERI, 2017, p.46), faz parte da proposta deste projeto o desenvolvimento de um plano de Placemaking Criativo e Tático, visando uma aprendizagem ativa.

A intervenção experimental em espaço público aqui proposta foi planejada de acordo com os 11 princípios fundamentais delineados pela organização não governamental americana Project For Public Spaces (PPS) para uma intervenção e transformação do tipo Placemaking. São estas as etapas propostas para o desenvolvimento do projeto de intervenção: 
- Envolvimento com as pessoas da comunidade: Identificação dos talentos e de pessoas interessadas pelo espaço; montagem de equipe multidisciplinar e voluntária, com a realização de pesquisa iconográfica e qualitativa; leitura visual do lugar, a fim de se analisar os significados cromáticos, extraindo-se uma paleta cromática à luz da cartografia cromáticogeográfica proposta por Jean Paulo Lenclos (LENCLOS, 1982 apud BOERI, 2017, p. 58-59), com a observação das cores permanentes e impermanentes; análise de experiências de Placemaking anteriores e de outras iniciativas, diversificadas de ações de Placemaking, para o entendimento da dimensão criativa da cor naquele local (Boeri, 2017).

- Pesquisa de observação do espaço e de seus usuários: Pesquisa iconográfica e de observação; focus group.

- Visão para o espaço, com entendimento da identidade local: Execução de workshops de subjetividade e identidade de cor com as crianças, jovens, adultos, artistas e entusiastas da comunidade, para a elaboração de um painel semântico da região; a partir do resultado obtido nesses workshops, avançar para a construção de uma paleta cromática a partir do "método de entendimento das cores subjetivas" (GALEOTTI, 2018).

- Triangulação: Posicionamento de equipamentos públicos próximos uns aos outros e verificação de resultados; análise e possível remanejamento; utilização de Realidade Aumentada e Realidade Virtual para na verificação, ademais da observação in loco.

- Elaboração de um projeto de intervenção de microescala, simples, rápido e de baixo custo: Verificação da pertinência do uso das tecnologias de Realidade Virtual e Realidade Aumentada.

- Criação de dois a três projetos cromáticos para serem aplicados no projeto final de Placemaking Criativo: Utilização das cores identificadas nas etapas anteriores; uso das tecnologias de VR e AR para apresentar a solução para a comunidade, a fim de que façam uma avaliação da paleta cromática com a qual se identificam; ao final, se possível, executar o projeto na região (ou seja, entende-se que a utilização das tecnologias de VR e AR é uma estratégia de baixo custo: as cores serão aplicadas em um ambiente 3D em que as pessoas poderão visualizar o resultado e manifestar sua preferência). 
Bases para desenvolvimento de um projeto cromático como intervenções de melhoria em áreas urbanas degeneradas usando tecnologias de Realidade

Virtual e Realidade Aumentada

- Elaboração de um relatório de trabalho

- Elaboração das bases para desenvolvimento de um projeto cromático urbano para intervenções de Placemaking Tático e Criativo com o uso de tecnologia

A criação de bases para o desenvolvimento de projetos cromáticos urbanos em áreas degeneradas se justifica pela falta de conhecimento teórico e prático no uso da cor enquanto elemento de linguagem e significado. Tal tema é uma questão recorrente nas discussões do Study Group on Colour Education, grupo de pesquisa liderado por Robert Hirschler e pertencente à Associação Internacional da Cor (AIC), assim como em outras entidades que têm a cor como objeto de estudo e pesquisa.

Portanto, este projeto pretende ser, além de uma solução projetual de design urbano para espaços públicos degenerados, uma forma de compreender e sistematizar a aplicação da cor com fins de resgatar a identidade de um lugar. Com isso, ele almeja enriquecer as soluções e oferecer as bases para o desenvolvimento de um projeto cromático urbano que melhor traduza tal identidade, a fim de promover o senso de lugar e de comunidade e, principalmente, devolver e expandir o senso de pertencimento da área em questão.

\section{Referências Bibliográficas}

ATEVERSA, Mônica Nador: Arte, comunidade, transformação. UFRGS, Rio Grande do Sul. Disponível em: https://www.ufrgs.br/arteversa/?p=735. Acesso em: 13 de março de 2019. BOA MISTURA. Cinco Cabezas, Diez manos, um solo corazón. Portfolio, 2019. Disponível em: https://bit.ly/2WoXyUX. Acesso em: 13 de março de 2019.

BOERI, C. Color loci. Placemaking: The urban color between needs of continuity and renewal. Color Res Appl. 2017; 42:641-649. https://doi.org/10.1002/col.22128.

CESAR, J. C. de O. O NCS - Natural Colour System e possíveis aplicações no projeto arquitetônico. São Paulo, 2010. Disponível em https://bit.ly/2WqHQIM.

COATES, Gary J.; SEAMON, David. Toward a Phenomenology of Place and Place-Making: Interpreting Landscape, Lifeworld and Aesthetics. Oz, 1984. Vol. 6. https://doi. org/10.4148/2378-5853.1074.

CSILLAG, P. et al. Overview of Color Education in Brazilian Universities: a Focus on Design Courses in the State of São Paulo. Journal of the International Colour Association, 2018: 22, 4-14. Disponível em http://www.aic-colour.org/journal.htm.

MCMILLAN, David W.; CHAVIS, David M. Sense of Community: A Definition and Theory. Journal of Community Psychology Volume 14, - George Peabody College of Vanderbilt University. January, 1986. Disponível em https://bit.ly/2n4DKoS.

DURÃO, M. J. Colour and Space: An Analysis of the Relationships between Colour Meaning Expression and the Perception of Space. Unpublished Ph.D. thesis, Universidade de Salford, Manchester, Inglaterra, 2000. 
Bases para desenvolvimento de um projeto cromático como intervenções de melhoria em áreas urbanas degeneradas usando tecnologias de Realidade

Virtual e Realidade Aumentada

GAGE, J. Colour and Meaning: Art, Science and Symbolism. London: Thames and Hudson, 2002. HARD, A. et al. NCS, Natural Calor System: From Concept to Research and Applications. Part 1. Color Reseorch ond Application, 21 (3). June. pp.180- 205. New York: John Wiley and Sons, 1996

HEEMANN, J; SANTIAGO, P.C. Guia do espaço público para inspirar e transformar. Placemaking Brasil, 2012. Disponível em https://bit.ly/1G4FUvP. Acesso em: 13 de março de 2019. HIRSCHLER, R. JAIC. Special Issue on Colour Education. Journal of the International Colour Association. 2018: 22, 1-3. Disponível em https://bit.ly/31l6q1p. Acesso em: 13 de março de 2019.

HIRSCHLER, R. Teaching colour to non-scientists. ICA-B Colour Symposium 2019 - Connecting colour in design, art \& science, Ghent, 2019.

ITTEN, J. The Art of Color: The Subjective Experience and Objective Rationale of Color. New York: Reinhold Publishing Corporation, 196.

KOBAYASHI, S. Color Image Scale. Tradução: Louella Matsunaga. 1st ed. London: Kodansha International Ltd., 1991.

LENCLOS, J. Colors of the World: a geography of Colour. W.W. Norton. New York, 2004.

LEW, A. A. Tourism Planning and Place Making: PlaceMaking or Placemaking?. Tourism Geographies 19:3, 448-446. Disponível em https://bit.ly/2WxV2AH. Acesso em: 13 de março de 2019.

LYDON, M.; BARTMAN, D.; GARCIA, T.; PRESTON, R.; WOUDSTRA. Urbanismo Tático: Ação de Curto Prazo, Mudanças de Longo Prazo. Vol.1/Vol2. The Street Plans Collaborative. Disponível em https://bit.ly/2IrIP6D. Acesso em: 13 de março de 2019.

MARICONDE, M. M.A; FERRARIS, R.; BALIÁN, M.; BARBOZA, M.; FERRARIS, V.; GIRELLI, I.; KENNY, S.; OJEDA, B.; SUAREZ, D.; SUEZ, L. Ideas de color: Acciones cromáticas urbanas desde la enseñanza. 2018.

MATHEU, J.C.I. World Habitat Day 2015. Message of the UN-Habitat Executive Director. MAZZILLI, C. T. S. A identidade cromática do ambiente urbano, em projeto do lugar: Colaboração entre psicologia, arquitetura e urbanismo. Rio de Janeiro - PROARQ, 2002.

MAZZILLI, C. T. S. Colour, City and Visual Narrative. In: AIC 2016 Interim Meeting: Color in Urban Life, 2016, Santiago. AIC2016 Interim Meeting, Color in Urban Life: Images, Objects and Spaces. Book Of Proceedings. Santiago: Asociación Chilena del Color, 2016. v. 1. p. 297-301.

MAZZILLI, C. T. S. Processos experimentais e linguagem em design visual. In: $5^{\circ}$ encuentro BID centros iberoamericanos de enseñanza de diseño, 2013, Madri. 5ำ encuentro BID centros iberoamericanos de enseñanza de diseño. Madrid: Central de Diseño - Matadero Madrid, 2013. v. 1. p. 52-54.

MAZZILLI, C. T. S.; CAETANO, L. M.; ARMENTANO, I. M. D.; SOUTO, I. C. S.. Environmental visual language: Teaching and research in Design-Art-Architecture interface. In: 2nd International Conference on Environmental Design, 2017, Turim. Proceedings of the 2nd International Conference on Environmental Design. Milão: De Lettera Publisher, 2017. v. 1. p. 39-52. MCMAHON, E. T. The Place Making Dividend: Diary of Commissioners for Planning / Number 80, p.17, 2010.

ORGANIZAÇÃO DAS NAÇÕES UNIDAS. Placemaking and the future of cities. Project for Public Spaces, 2012. 
Bases para desenvolvimento de um projeto cromático como intervenções de melhoria em áreas urbanas degeneradas usando tecnologias de Realidade Virtual e Realidade Aumentada

OZEL, BILGE, MECCA, SAVERIO. Rethinking the Role of Public Spaces for Urban Resilience: Case Study of Eco-village in Cenaia. Past Present and Future of Public Space $Đ$ International Conference on Art, Architecture and Urban Design, Bologna (Italy), June 25 -27, 2014. PEIXOTO, P.; PROVIDÊNCIA, P.; AGUIAR, J. A cor como linguagem: reflexões sociológicas sobre as dinâmicas cromáticas nos revestimentos e acabamentos históricos de Coimbra in LNEC (org.), Património em construção: contextos para a sua preservação. Lisboa, 2011: LNEC, 247-254.

PROJECT FOR PUBLIC SPACES. Placemaking and the future of cities. Draft, 2012. Disponível em https://bit.ly/2X3mFkN. Acesso em: 27 de maio, 2019.

SUÁREZ, D.; BALIÁN, M. et al. The role of environmental colour in the experience and identity of the city. (pp. 209-213). Proceedings of the AIC 2018 - Interim Meeting of the International Colour Association "Color \& human confort". Lisboa, 2018.

SUÁREZ, D.; INCATASCIATO, A. et al. The Chromatic Project as a proposal to reappraise the Urban image, Co-management experience between the Municipal Government and the University. (pp. 738-74). Proceedings of the AIC 2014 - Interim Meeting of the International Colour Association "Color, culture and identity: past, present and future". México, 2014.

SUÁREZ, D.; Zoppi, C. Colour as a designer of an urban place: patrimony, identity and tourism in the city of Cordoba, Argentina. (pp. 613-617). Proceedings of the AIC 2018 - Interim Meeting of the International Colour Association "Color \& human confort". Lisboa, 2018. WEBB, D. Placemaking and social equity: expanding the framework of creative placemaking. Seattle University Artivate: A Journal of Entrepreneurship in the Arts Volume 3, Issue 1 pp. 35-48,2013. Dsiponível em http://artivate.org. Acesso em: 27 de maio, 2019.

WORLD COMMISSION ON ENVIRONMENT AND DEVELOPMENT. (WCED) Our Common Future. United Nations WCED, Oxford University Press, Oxford, U.K. 1987.

WYCKOFF, M.A. Definition of Placemaking: Four Different Types. Planning and Zoning News. 2014. Tourism planning and place making: place-making or placemaking. Disponível em http://pznews.net/media/. Acesso em: 27 de maio, 2019.

ZAKARIYA K, MOHYUDDIN, A.; YAMAN, M. Refining Tourist's Place Experience through Placemaking: Concepts and Correlations. The International Journal of Diversity in Organizations, Communities and Nations.Vol.7 p. 249-257, 2007.Disponível em https://bit.ly/31kvcPy. 